The Proceedings of the Nutrition Society, published by Cambridge University Press, in part record meetings of the Symposium type, at which experts in a particular field are invited by Council to make contributions on specific parts of it and at which general discussion follows these invited contributions. The meetings also include sessions at which papers are communicated by members and others on original work. It is proposed at present to publish summaries of the papers read at each meeting, each communication being recorded in the Society's Proceedings by means of an abstract not exceeding in length 400 words or the equivalent space in print. The Proceedings are published three times a year.

The subscription (excluding VAT) to the Proceedings is $£ 117.00$ net (US $\$ 229.00$ in USA, Canada and Mexico). Single issues are $£ 41.00$ (US $\$ 79.00$ in the USA, Canada and Mexico) each; postage extra.

Copying. This journal is registered with the Copyright Clearance Center, 27 Congress Street, Salem, Mass. 01970. Organizations in the USA who are also registered with C.C.C. may therefore copy material (beyond the limits permitted by sections 107 and 108 of the US copyright law) subject to payment to C.C.C. of the per-copy fee of \$5.00. This consent does not extend to multiple copying for promotional or commercial purposes. Code $0007-1145 / 94 \$ 5.00+.00$. Organizations authorized by the Copyright Licensing Agency may also copy material subject to the usual conditions.

ISI Tear Sheet Service, 3501 Market Street, Philadelphia, Pennsylvania 19104, USA, is authorized to supply single copies of separate articles for private use only.

For all other use, permission should be sought from Cambridge or the American Branch of Cambridge University Press.

\begin{tabular}{|c|c|c|}
\hline Andersen, L. M. & 345 & Galan, Pilar \\
\hline Baigent, D. R. & 345 & Hansen, Carsten \\
\hline Balle, Mette & 449 & Hauerslev, Catrine \\
\hline Barber, Teresa & 361 & Hautvast, G. A. J. \\
\hline Batterham, E. S. & 345 & Hercberg, Serge \\
\hline Belleville, Jacques & 375 & Hermansen, Kjeld \\
\hline Boekholt, Harry A. & 335 & Hurrell, Richard F. \\
\hline Bouziane, Mahmoud & 375 & Hynd, P. I. \\
\hline Cabo, José & 361 & Kabir, Nazma \\
\hline Chapman, R. E. & 425 & Kastenmayer, Peter \\
\hline Cherouvrier, Françoise & 411 & \\
\hline Christiansen, Christian & 449 & $\begin{array}{l}\text { MacDonald, I. A. } \\
\text { Martin, G. B. }\end{array}$ \\
\hline $\begin{array}{l}\text { Davidsson, Lena } \\
\text { de Groot, Lisette C. P. G. M. }\end{array}$ & $\begin{array}{l}411 \\
335\end{array}$ & Morgan, Linda M. \\
\hline Deurenberg, Paul & 309 & Pettersson, D. \\
\hline Drijvers, José J. M. M. & 335 & Prost, Josiane \\
\hline Estornell, Ernesto & 361 & Quinlan, P. T. \\
\hline Forsum, Elisabet & 317 & Rasmussen, Ole \\
\hline
\end{tabular}

\title{
INDEX OF AUTHORS
}

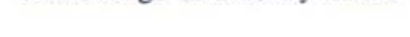

Forsum, Elisabet

\begin{tabular}{l|lr}
411 & Razdan, A. & 389 \\
449 & Sadurskis, Aija & 317 \\
449 & Schouten, Frans J. M. & 335 \\
335 & Sohlström, Annica & 317 \\
411 & Southgate, D. A. T. & 307 \\
449 & Spaaij, Caroline J. K. & 335 \\
411 & & \\
425 & Thomsen, Claus & 449 \\
& & \\
317 & van Raaij, Joop M. A. & 335 \\
411 & van der Heijden, Lidwien J. M. \\
& & 335 \\
437 & Velthuis-Te Wierik, Erica J. M. \\
425 & & 309 \\
401 & & \\
& & 437 \\
389 & Webber, J. & 309 \\
375 & Westerterp, Klaas R. & 425 \\
& White, C. L. & 401 \\
401 & Williams, Christine M. & 401 \\
& Wright, J. & 401 \\
449 & Zampelas, A. &
\end{tabular}




\section{British Journal}

\section{of Nutrition}

VOL. 71 NO. 3 MARCH 1994

\section{CONTENTS}

\section{Editorial}

Directions from Contributors. D. A. T. Southgate

Comparison of methods for measuring body composition

Between-laboratory comparison of densitometry and bio-electrical impedance measurements. Paul Deurenberg, Klaas R. Westerterp \& Erica J. M. Velthuis-Te Wierik

Effects of energy restriction on body composition in pregnant rats

Body composition and fat distribution during the first 2 weeks of gestation in ad lib.-fed and energy-restricted rats. Annica Sohlström, Nazma Kabir, Aija Sadurskis \& Elisabet Forsum

Dietary induced thermogenesis in pregnancy

No substantial reduction of the thermic effect of a meal during pregnancy in wellnourished Dutch women. Caroline J. K. Spaaij, Joop M. A. Van Raaij, Lidwien J. M. Van Der Heijden, Frans J. M. Schouten, José J. M. M. Drijvers, Lisette C. P. G. M. De Groot, Harry A. Boekholt \& Joseph G. A. J. Hautvast

Utilization of tryptophan derived from ileal digestion

Utilization of ileal digestible amino acids by growing pigs: tryptophan. E. S. Batterham, L. M. Andersen \& D. R. Baigent

Interactions between nitrogen and lipid metabolism

Improved nitrogen metabolism in rats fed on lipid-rich liquid diets. Ernesto Estornell, Teresa Barber \& José Cabo

Effects of dietary composition and frequency on lipid metabolism

Changes in fatty acid compositions of total serum and lipoprotein particles, in growing rats given protein-deficient diets with either hydrogenated coconut or salmon oils as fat sources. Mahmoud Bouziane, Josiane Prost \& Jacques Belleville

Effects of feeding restriction and meal pattern of a sugar beet-containing diet and control diet on nutrient digestibility, plasma lipid concentrations and postprandial triacylglycerol response in broiler chickens. A. Razdan \& D. Pettersson

Effects of triacylglyceride positional structure on lipid metabolism

The effect of triacylglycerol fatty acid positional distribution on postprandial plasma metabolite and hormone responses in normal adult men. A. Zampelas, Christine M. Williams, Linda M. Morgan, J. Wright \& P. T. Quinlan 